\title{
Impact of Downlink Distributed and Adjacent Subcarrier Permutation Modes on the Performance of Mobile WiMAX System in ITU-R Ped. B Channel with Obtaining Optimum Cyclic Prefix
}

\section{Safwan E. Abdul Fatah}

\section{Dr. Sami A. Mawjoud}

\section{Abstract}

Mobile WiMAX is one of the candidate technologies for $4 \mathrm{G}$ wireless systems, promising high data rates and affluent multimedia services. The mobile WiMAX standard (IEEE802.16e-2005)is based on Orthogonal Frequency Division Multiple Access (OFDMA), which allows a very efficient use of bandwidth in a wide frequency range, this is due to the fact that OFDMA uses multi-channel OFDM approach and provides subcarrier access in the time and the frequency domains.

In this paper the impact of downlink distributed (FUSC, PUSC) and adjacent (AMC) subcarrier permutation modes used in mobile WiMAX system are investigated using simulation under various modulation and coding schemes (link speeds)with the support of optimum value of the Cyclic Prefix (CP) for the OFDMA symbol duration. The results are expressed in terms of the maximum achievable throughput and operating distance from base station in pedestrian multipath fading channel (ITU-R Ped. B at $3 \mathrm{Km} / \mathrm{h}$ speed) under a UDP-based application (video streaming), which is the proper application for multimedia services. The results show the optimum cyclic prefix valve for the FUSC, PUSC and AMC subcarrier permutation modes to achieve the best system performance, also the results show differences in PER performance atvarious modulation and coding schemes for the three subcarrier permutation modes (FUSC, PUSC and AMC). These differences in performance are exploited in the present work to show the best threshold SNR to switch from link speed to another link speed in terms of throughput and operating distance.

Key words: IEEE802.16e-2005, OFDMA, FUSC, PUSC, AMC, ITU-R Ped. B channel, PER.

$$
\text { تأثير تباديل الحاملات الفرعية الموزعة والمتجاورة للاتجاه النازل على اداء نظام الواد الواي }
$$

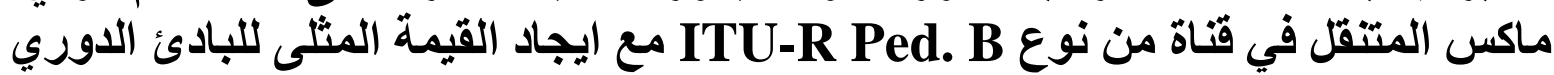

$$
\text { د. سامي عبدالموجود }
$$

$$
\text { صفوان عماد عبد الفتاح }
$$

يعتبر نظام الواي ماكس المتنقل احد انواع التكنلوجيا المرشحة للجيل الرابع اللاسلكي والذي يتميز بمعدل نقل بيانات

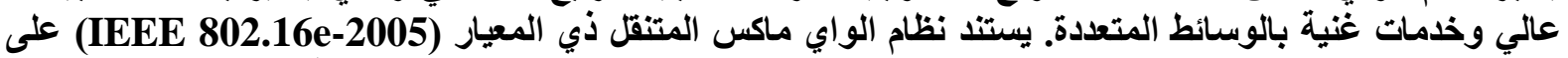

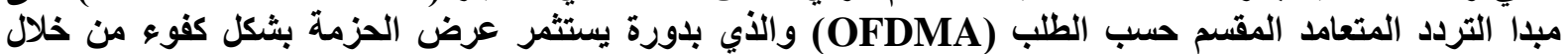

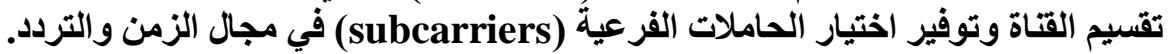

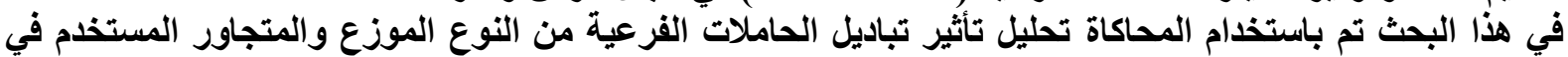

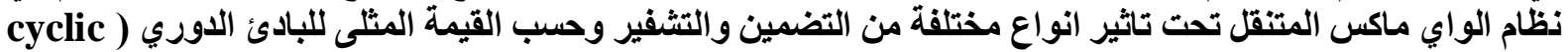

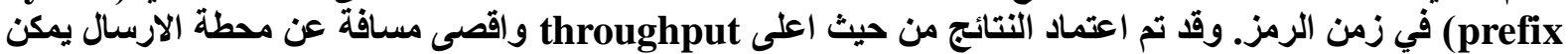

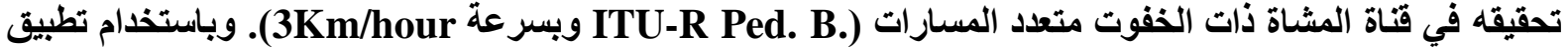

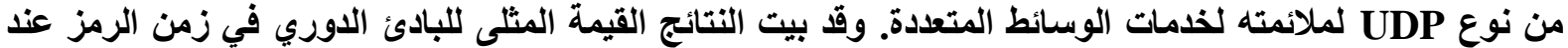

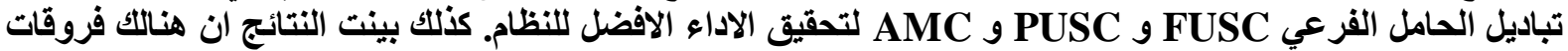

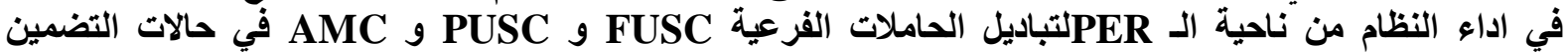
وألتثفير المختلفة، حيث تم استثمارها في هذا العمل لإيجاد افضل قيمة SNR للتحويل من سرعة ارتباط الى الى اخرى من حيث الـ throughput ومسافة العمل. 


\section{1- Introduction:}

Wireless Metropolitan Area Network (Wireless MAN) or worldwide interoperability for Microwave Access (WiMAX), which is mainly based on the IEEE standard is the leading technology for delivering wireless internet services and offers high-throughput broadband connections over long distances. In December 2005, the IEEE group completed and approved IEEE 802.16e-2005, an amendment to the IEEE 802.16-2004 standard that added mobility support. The IEEE 802.16e-2005 forms the basis for the WiMAX solution for nomadic and mobile applications and is often referred to as mobile WiMAX [1,2,3].The Mobile WiMAX air interface adopts Orthogonal Frequency Division Multiple Access (OFDMA) for improved multi-path performance in non-line-of-sight environments. OFDMA is a flexible multipleaccess technique that can accommodate many users with widely varying applications, data rates, and QoS requirements [3].Scalable OFDMA (SOFDMA) [4] is introduced in the IEEE 802.16e-2005 Amendment to support scalable channel bandwidths (typically $1.25 \mathrm{MHz}, 5$ $\mathrm{MHz}, 10 \mathrm{MHz}$ or $20 \mathrm{MHz}$ ). The scalability is supported by scaling the Fast Fourier transforms (FFT) size to the channel bandwidth in order to fix the subcarrier frequency spacing at $10.94 \mathrm{kHz}$ [5]. The SOFDMA parameters are listed in Table (3).

When designing SOFDMA wireless systems the optimum choice of the number of subcarriers per channel bandwidth is a tradeoff between protection against multipath, Doppler shift, and design cost/complexity. When the time delay between the various signal paths is a significant fraction of the transmitted signals symbol period, a transmitted symbol may arrive at the receiver during the next symbol period and cause intersymbol interference (ISI). At higher data rates, the symbol time is shorter; hence, it takes only a smaller delay to cause ISI [3]. Calculations in [4] show that the optimum tradeoff for mobile systems is achieved when subcarrier spacing is $10.94 \mathrm{kHz}[5,6]$.

Inverse-Fast-Fourier-Transforming (IFFT) creates the OFDMA symbol time $\left(T_{s}=T_{b}+T_{g}\right)$. Time duration $T_{b}$ is referred to as the useful symbol time. Guard Time (GT) length is one of the key OFDMA parameters. Termed Cyclic Prefix (CP), a copy of the last $T_{g}$ of the useful symbol period appended to the beginning of each transmitted symbol to completely suppress ISI as long as the GT is greater than the channel delay spread. Besides it is used to collect the multipath signals, it is essential to maintain the orthogonally of the subcarriers $[1,2]$.

Also by implementing the $\mathrm{GT}$ as a $\mathrm{CP}$ the system being immune to Intermarried Interference (ICI) which causes a severe degradation of Quality of Service (QoS) in OFDMA systems [7,8]. Adding a guard interval, however, implies power wastage and a decrease in bandwidth efficiency. The amount of power wasted depends on how large a fraction of the OFDMA symbol duration the guard time is. Therefore, the larger the symbol period leading to smaller loss in power and bandwidth efficiency [3].

An OFDMA symbol is made up of subcarriers, the number of which determines the FFT size used. In the frequency domain, each OFDMA symbol is created by mapping the sequence of symbols on the subcarriers. WiMAX has three classes of subcarriers.

1. Data subcarriers are used for carrying data symbols.

2. Pilot subcarriers are used for carrying pilot symbols. The pilot symbols are known a priori and can be used for channel estimation and channel tracking.

3. Null subcarriers have no power allocated to them, including the DC subcarrier and the guard subcarriers toward the edge. The DC subcarrier is not modulated, to prevent any saturation effects or excess power draw at the amplifier. No power is allocated to the guard subcarriers toward the edge of the spectrum in order to fit the spectrum of the OFDMA symbol within the allocated bandwidth and thus reduce the interference between adjacent channels. 
In the OFDMA mode, the active subcarriers are divided into subsets of subcarriers, each subset is termed a sub channel, and here, sub channels form the minimum frequency resource-unit allocated by the Base Station (BS). Therefore, different sub channels may be allocated to different users as a multiple-access mechanism. This type of multi-access scheme gives the (OFDMA) its name. In the downlink (DL), a sub channel may be intended for different (groups of) receivers; in the uplink (UL), a transmitter may be assigned one or more sub channels, several transmitters may transmit simultaneously. The subcarriers forming one sub channel may, but need not be adjacent as illustrated in figure (1).

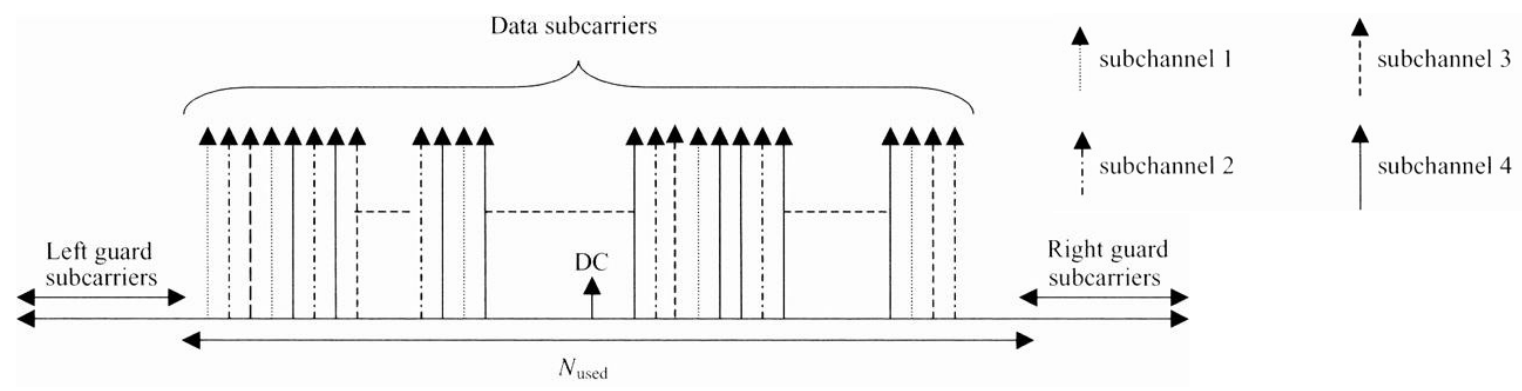

Figure (1): OFDMA frequency description (4 sub channel schematic example)

The WiMAX physical layer is also responsible for slot allocation and framing over the air. The minimum time-frequency resource that can be allocated by a WiMAX system to a given link is called a slot $[2,3]$.

In IEEE 802.16e-2005, both Frequency Division Duplexing (FDD) and Time Division Duplexing (TDD) are allowed. Most WiMAX deployments are likely to be in TDD mode because of its advantages, which are: TDD allows for a more flexible sharing of bandwidth between uplink and downlink, does not require paired spectrum, has a reciprocal channel that can be exploited for spatial processing, and has a simpler transceiver design. WiMAX is quite flexible in terms of how multiple users and packets are multiplexed on a single frame. A single downlink frame may contain multiple bursts of varying size and type carrying data for several users. The frame size is also variable on a frame-by-frame basis from $2 \mathrm{~ms}$ to $20 \mathrm{~ms}$ [3].

\section{2-Subcarrier Permutation Modes in Mobile WiMAX OFDMA Physical Layer:}

Mobile WiMAX based on OFDMA allows several modes of subcarrier permutation, which are described in [1, 2, 3, 9, and 10]. Sub channels may be constituted using either contiguous subcarriers or subcarriers pseudo-randomly distributed across the frequency spectrum. Two families of distribution modes can be distinguished:

- Diversity (or distributed) permutations: The subcarriers are distributed pseudorandomly, this family includes: FUSC (Full Usage of the Sub Channels) and PUSC (Partial Usage of the Sub Channels), OPUSC (Optional PUSC), OFUSC (Optional FUSC) and TUSC (Tile Usage of Sub Channels). The main advantages of distributed permutations are frequency diversity and intercell interference averaging. Diversity permutations minimize the probability of using the same subcarrier in adjacent sectors or cells. On the other hand, channel estimation is not easy as the subcarriers are distributed over the available bandwidth.

- Contiguous (or adjacent) permutations: These consider a group of adjacent subcarriers. This family includes the AMC (Adaptive Modulation and Coding) mode. Although frequency diversity is lost, AMC allows system designers to exploit multiuser diversity, allocating sub channels to users based on their frequency response. Multiuser 
diversity can provide significant gains in overall system capacity, if the system strives to provide each user with a sub channel that maximizes its received SINR.

Each DL sub frame and UL sub frame in IEEE 802.16e-2005 is divided into various zones, each using a different subcarrier permutation mode. The OFDMA frame may include multiple zones (such as PUSC, FUSC, optional FUSC, AMC, TUSC1, and TUSC2). The maximum number of DL zones is eight in one DL sub frame. The physical layer parameters may change from one zone to the next. More than one DL or UL zone may be defined for each configuration. Figure (2) depicts the OFDMA TDD frame with multiple zones [1,2].

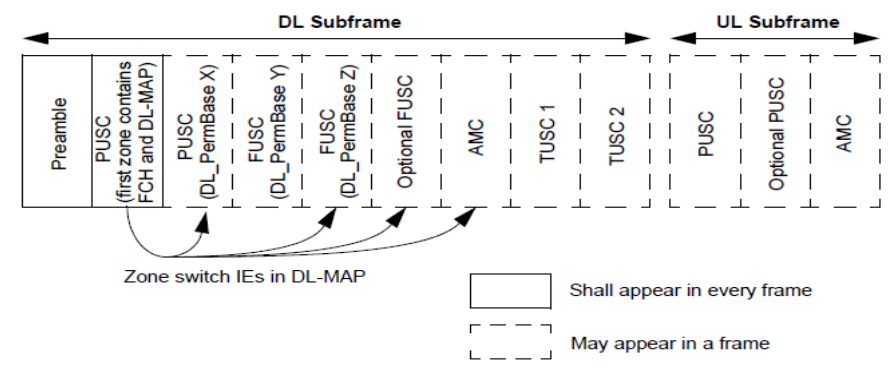

Figure (2): Illustration of OFDMA TDD frame with multiple zones

Mandatory permutation modes of the presently defined mobile WiMAX profiles are:

- For the downlink: PUSC, FUSC and AMC;

- For the uplink: PUSC and AMC.

In this paper, we will concentrate on the downlink mandatory permutation modes PUSC, FUSC and AMC.

\subsection{Downlink Full Usage of Sub channels (FUSC) Permutation Mode:}

In the case of DL FUSC, all the data subcarriers are used to create the various subchannels. Each

Sub channel is made of 48 data subcarriers, which are distributed evenly throughout the entire frequency band.

In FUSC, the pilot subcarriers are allocated first, and then the remainder of the subcarriers are mapped onto the various sub channels, using a permutation scheme $[3,4]$. After mapping all pilots, the remainder of the used subcarriers is used to define the data sub channels. To allocate the data sub channels, the remaining subcarriers are partitioned into groups of contiguous sub carriers. Each sub channel consists of one subcarrier from each of these groups. The number of groups is therefore equal to the number of subcarriers per sub channel, and it is denoted $N_{\text {subcarriers }}$. The number of the subcarriers in a group is equal to the number of sub channels, and it is denoted $N_{\text {subchannels. }}$. As already mentioned, the number of data subcarriers in every sub channel is the same $N_{\text {subcarriers }}=48$. With 1024 -FFT, there are 16 sub channels in total. The number of data subcarriers is thus equal to $N_{\text {subcarriers }} \times N_{\text {subchannels }}=48 \times 16=768$ data subcarrier.

During subcarrier distribution process, one subcarrier is picked up using the permutation formula [1,2] from every group and is being allocated to a sub channel. Figure (3) is used to clarify the subcarrier permutation process for DL FUSC. 


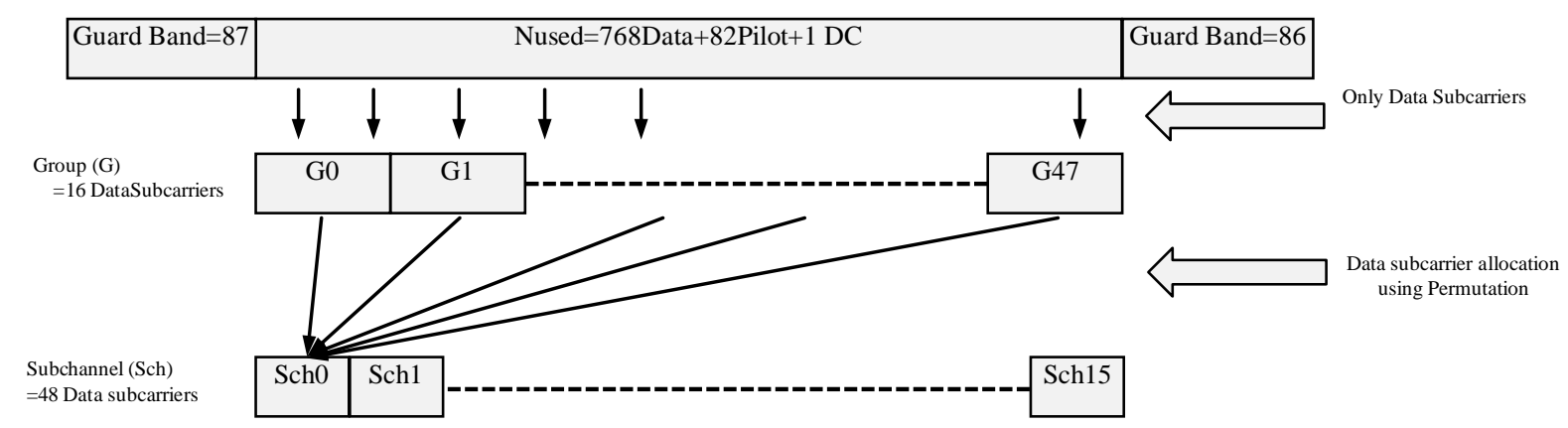

Figure (3): Distributed permutation process of DL FUSC (1024-FFT)

\subsection{Downlink Partial Usage of Sub channels (PUSC) Permutation Mode:}

PUSC is similar to FUSC except that all the subcarriers are first divided into six groups. Permutation of subcarriers to create sub channels is performed independently withineach group, thus, in essence, logically separating each group from the others.

One slot of PUSC is two OFDMA symbols by one sub channel, while one PUSCsubchannel comprises 24 data subcarriers. In the case of PUSC, all the subcarriers except the null subcarrier are first arranged into clusters. Each cluster consists of 14 adjacent subcarriers over two OFDMA symbols. In each cluster, the subcarriers are divided into 24 data subcarriers and 4 pilot subcarriers. The clusters are then renumbered using a pseudorandom numbering scheme, which in essence redistributes the logical identity of the clusters. After renumbering, the clusters are divided into six groups, with the first one-sixth of the clusters belonging to group 0, and so on. A sub channel is created using two clusters from the same group.

Some useful terms specific to the DL PUSC:

Physical Cluster: It is a set of 14 adjacent subcarriers (12data+2pilot). These clusters are contiguous in the frequency band.

Logical Cluster: Physical Clustered are renumbered according to a renumbering sequence to form logical clusters. This renumbering sequence is given in $[1,2]$. The two adjacent logical clusters are not contiguous in frequency band.

Group: It is a set of logical clusters. These groups are commonly referred as major groups. There are six groups in total. With 1024-FFT, the even group consists of 12 logical clusters while the odd group comprises 8 logical clusters. For the purpose of inner permutation, the subcarriers of each group are indexed separately, i.e., every group will have subcarriers index starting from ' 0 '.

Outer Permutation: It is the process by which physical clusters are renumbered to give rise to logical clusters.

Inner Permutation: The process to form sub channels from the subcarrier of logical clusters of a group is called inner permutation.

The permutation process to form sub channels in DL PUSC is shown in figure (4).

Pilot positions are marked separately for odd and even OFDMA symbol. These subcarriers will be separated from the rest before inner permutation. The pilot subcarriers for the odd symbols will be ' 4 ' and ' 8 ', the pilot subcarriers positions for the even symbols will be ' 0 ' and ' 12 '. The rest twelve subcarriers will be used as data subcarriers in the inner permutation.

In PUSC, it is possible to allocate all or only a subset of the six groups to a given transmitter. By allocating disjoint subsets of the six available groups to neighboring transmitters, it is possible to separate their signals in the subcarrier space, thus enabling a tighter frequency reuse at the cost of data rate. Such usage of subcarriers is referred to as segmentation. 


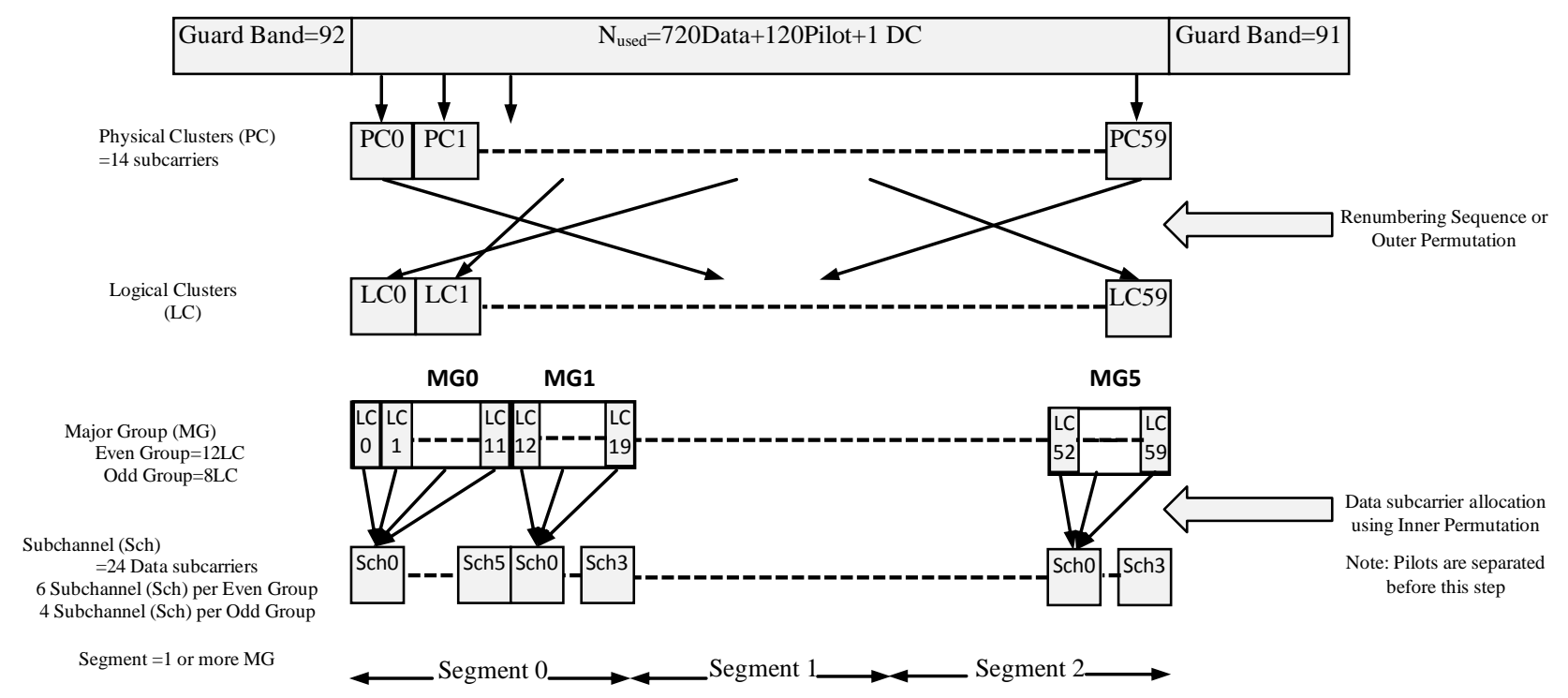

Figure (4): Distributed permutation process of DL PUSC (1024-FFT)

\subsection{Downlink Adaptive Modulation and Coding (AMC) Permutation Mode:}

Unique to the AMC permutation mode, all subcarriers constituting a subchannel are adjacent to each other. In this subcarrier permutation, nine adjacent subcarriers with eight data subcarriers and one pilot subcarrier are used to form a bin.

Some useful definitions in the AMC:-

Bin: It is the smallest unit in frequency domain for adjacent subcarrier permutation. It is composed of 9 contiguous subcarriers. Out of 9, eight are data tones and one is pilot tone.

Slot: There are four different slot structures types. In the first type (default type), a slot is six contiguous bins by one symbol. The second typeis two bins by three symbols. The third type is three bins by two OFDMA symbols and for the fourth and last type, a slot is one bin by six symbols. If $\mathrm{N}$ is number of bins and $\mathrm{M}$ is number of symbols then product $\mathrm{N} \times \mathrm{M}$ is always equal to six.

Sub channel: The sub channel in adjacent subcarrier permutation is composed of bins. Number of bins depends upon slot structure. For example for default type, a sub channel comprises six bins. For the rest of the three types, sub channel has two, three and one bins respectively.

Physical Band: A group of four adjacent bins in the frequency domain is called a physical band.

Logical Band: A logical band is a group of physical bands. For 1024-FFT, number of physical bands per logical band is two.

The permutation process to form subchannels in DL AMC is shown in figure (5). 
Abdul-Mawjoud: Impact of Downlink Distributed and Adjacent Subcarrier------

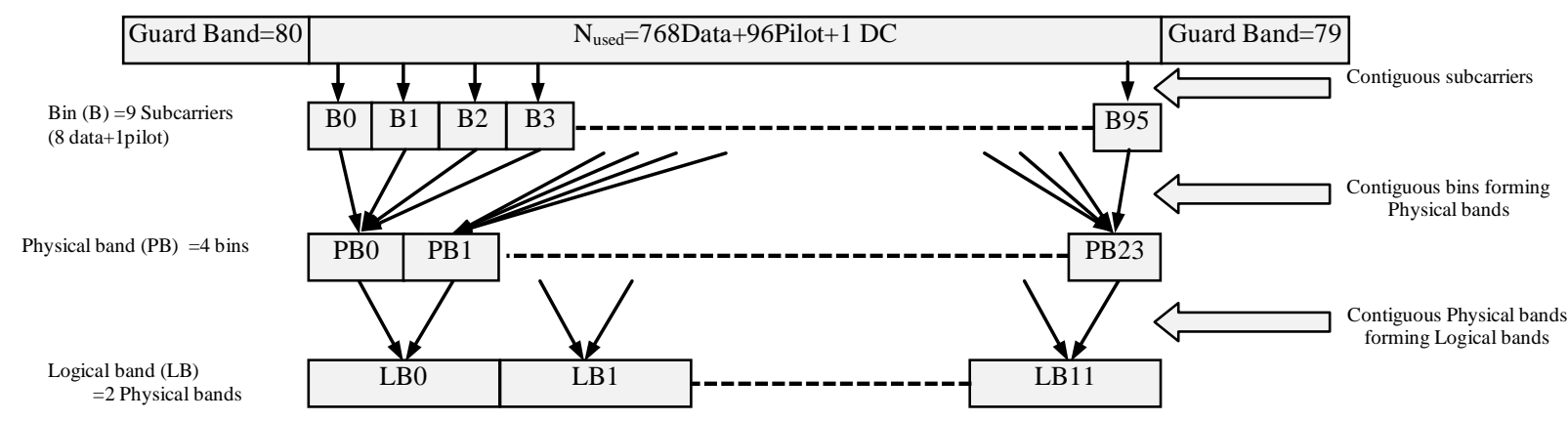

Figure (5): Adjacent permutation process of DL AMC (1024-FFT)

The various parameters related to the FUSC, PUSC, AMC permutation scheme in the physical layer for 1024-FFT sizes are shown in table (1)[1,2].

Table (1): 1024-FFT, 10MHz-BW OFDMA FUSC, PUSC, AMC subcarrier allocationparameters

\begin{tabular}{|c|c|c|c|}
\hline \multirow[t]{2}{*}{ Parameters } & \multicolumn{3}{|c|}{ Subcarrier Permutation Mode } \\
\hline & FUSC & PUSC & $\mathrm{AMC}$ \\
\hline Number of Guard Subcarriers, Left & 87 & 92 & 80 \\
\hline Number of Guard Subcarriers, Right & 86 & 91 & 79 \\
\hline $\begin{array}{l}\text { Number of Used Subcarriers (Nused) } \\
\text { (including all possible allocated pilots } \\
\text { and the DC subcarrier) }\end{array}$ & 851 & 841 & 865 \\
\hline Number of data subcarriers & 768 & 720 & 768 \\
\hline Number of pilot Subcarriers & 82 & 120 & 96 \\
\hline Number of sub channels & 16 & 30 & 48 \\
\hline A slot consists of & $\begin{array}{l}\text { 1Subchannel× } \\
\text { 1OFDMA } \\
\text { symbol } \\
\end{array}$ & $\begin{array}{l}\text { 1Subchannel× } \\
\text { 2OFDMA } \\
\text { symbols } \\
\end{array}$ & $\begin{array}{l}\text { 2bins } \times 3 \text { OFD } \\
\text { MA symbols }\end{array}$ \\
\hline $\begin{array}{l}\text { Number of data subcarriers in each } \\
\text { symbol per sub channel }\end{array}$ & 48 & 24 & 16 \\
\hline Number of data Subcarriers per slot & 48 & 48 & 48 \\
\hline Number of physical bands & - & - & 24 \\
\hline Number of bins per physical band & - & - & 4 \\
\hline Number of subcarriers per cluster & - & 14 & - \\
\hline Number of clusters & - & 60 & - \\
\hline Number of PUSC groups & - & 6 & - \\
\hline Number of sub channels per even group & - & 6 & - \\
\hline Number of sub channels per odd group & - & 4 & - \\
\hline
\end{tabular}

\section{3- The Proposed Channel Model:}

The empirical channel model that generally used for describing the mobile WiMAX system is the ITU-R channel model, which was developed according to the ITU-R M.1225 Recommendation [11]. This model is implemented in this paper for the simulation of an IEEE 80216e-2005(mobile WiMAX) system.

In order to model the ITU-R channel between a transmit and receive antennas as aSISO (Single in Single Out) multipath channel, the Recommendation ITU-R M.1225 [12] introduces a channel impulse response model based on a tapped-delay-line. The number of taps, the time delay relative to the first tap, the average power relative to the strongest tap, 
characterize the model and the Doppler spectrum of each tap, recall that each tap represents a path delayer.

Equation (1) represents the impulse response of a tapped-delay-line multipath channel, which is described by a discrete number of impulses:

$$
h(t, \tau)=\sum_{i=1}^{N} c_{i}(t) \delta\left(\tau-\tau_{i}\right)
$$

Where, $t$ indicates the time variable and captures the time variability of the impulse response of each multipath component modeled typically as Rayleigh fading, and $\tau$ refer to the rms values of the path delays associated with each multipath. The coefficients $c_{i}(t)$ refer to the Power Profiles(variance) of each path relative to the first path. The selection of the $N$ and delay values $\tau_{i}$ depends upon what is considered a significant level. The implementation of equation (1) as a tapped-delay-line multipath channel by a 4-tap model (as an example) is shown in figure (6).

As illustrated in $[3,12$, and 13] the multipath channel classified according to the delay spread variability. ITU-R M.1225 has specified two multipath profiles, A and B, for vehicular, pedestrian, and indoor channels. Channel B has a much longer delay spread than channel A and is generally accepted as a good representative of urban macro-cellular environment. Channel A, on the other hand, is accepted as a good representative of rural macro-cellular environment.

The simulation presented in this paper is based on the pedestrian B channel (referred to as Ped. B) Model, since it is suitable for broadband wireless communications, also, according to WiMAX Forum $[5,12,13]$, recommending that WiMAX simulations should use the ITU Pedestrian B $(3 \mathrm{~km} / \mathrm{h})$ and Vehicular A $(60 \mathrm{~km} / \mathrm{h})$ mobility profiles.

Table (2) shows the normalized power profiles for the pedestrian B test environment tappeddelay-line parameters. For each tap of the channels, three parameters are given: the time delay relative to the first tap, the average power relative to the strongest tap, and the Doppler spectrum of each tap.

Table (2): Pedestrian B channel tapped-delayline parameters

\begin{tabular}{|c|c|c|c|}
\hline \multirow{2}{*}{$\begin{array}{c}\text { Ta } \\
\mathrm{p}\end{array}$} & \multicolumn{2}{|c|}{$\begin{array}{c}\text { Pedestrian B (Ped.B) } \\
\text { Channel }\end{array}$} & $\begin{array}{c}\text { Doppler } \\
\text { spectru } \\
\mathrm{m}\end{array}$ \\
\cline { 2 - 3 } & $\begin{array}{c}\text { Relative } \\
\text { delay (ns) }\end{array}$ & $\begin{array}{c}\text { Average } \\
\text { power (dB) }\end{array}$ & \\
\hline 1 & 0 & 0 & Classic \\
\hline 2 & 200 & -0.9 & Classic \\
\hline 3 & 800 & -4.9 & Classic \\
\hline 4 & 1200 & -8.0 & Classic \\
\hline 5 & 2300 & -7.8 & Classic \\
\hline 6 & 3700 & -23.9 & Classic \\
\hline
\end{tabular}

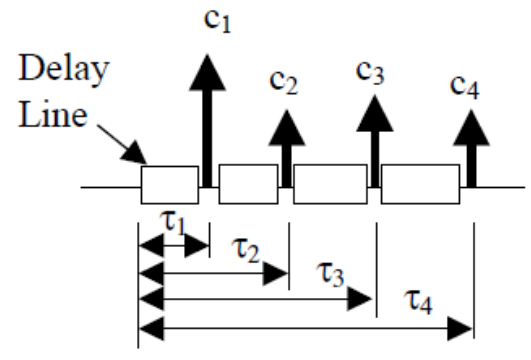

Figure (6): Tapped-delay-line model

\section{4- Simulation Parameters:}

link-level simulations are used to study the behavior of downlink performance for Mobile WiMAX system under multipath fading channel, the parameters defined in the physical layer are from IEEE standards [1,2], also [5] and [14], all the required physical layer parameters for simulation are listed in table(3).

The three subcarrier permutation modes(FUSC, PUSC, AMC) are based on Scalable OFDMA principle, features which are selected in our simulation are highlighted. The TDD 
duplexing has been chosen according to the advantages mentioned for reducing the simulation time; the frame duration of $2 \mathrm{msec}$. is selected. The convolutional turbo codes (CTC) is selected due to their superior performance over the other convolutional codes[3,7,11]. The Mobile Station (MS) Parameters such as velocity, max. delay spread, and max. Doppler spread as given in table (3) is selected according to the channel specification parameters.

Table (3): Mobile WiMAX system - physical layer simulation parameters

\begin{tabular}{|c|c|c|c|c|}
\hline \multicolumn{5}{|c|}{ OFDMA Parameters } \\
\hline Duplexing & \multicolumn{4}{|c|}{ TDD } \\
\hline Channel Bandwidth $(B W) \mathrm{MHz}$ & 1.25 & 5 & 10 & 20 \\
\hline FFT Size $\left(N_{F F T}\right)$ & 128 & 512 & 1024 & 2048 \\
\hline Sampling Factor ${ }^{*}(n)$ & $28 / 25$ & $28 / 25$ & $28 / 25$ & $28 / 25$ \\
\hline $\begin{array}{l}\text { Sampling Frequency } \mathrm{MHz}(F s=\text { floor }(n \times \\
B W / 8000) \times 8000)\end{array}$ & 1.4 & 5.6 & 11.2 & 22.4 \\
\hline $\begin{array}{l}\text { Subcarrier frequency Spacing }(\Delta f=F s / \\
\left.N_{F F T}\right)\end{array}$ & \multicolumn{4}{|c|}{$10.9375 \mathrm{kHz}$} \\
\hline Useful Symbol Time $\left(T_{b}\right) \quad\left(T_{b}=1 / \Delta f\right)$ & \multicolumn{4}{|c|}{$91.42 \mu \mathrm{sec}}$. \\
\hline $\begin{array}{l}\text { Cyclic Prefix }(\mathrm{CP}) \text { duration }\left(T_{g}=T_{b} / 32, T_{b}\right. \\
\left./ 16, T_{b} / 8, T_{b} / 4\right)\end{array}$ & \multicolumn{4}{|c|}{ Variable $(2.85,5.71,11.42,22.85) \mu$ sec. } \\
\hline OFDMA Symbol $\left(T_{s}=T_{b}+T_{g}\right)$ & \multicolumn{4}{|c|}{$\begin{array}{c}\text { Dependent on CP duration }(94.28,97.14,102.9, \\
114.28) \mu \mathrm{sec} .\end{array}$} \\
\hline Frame duration & \multicolumn{4}{|c|}{2 msec. } \\
\hline Number of OFDMA symbols & \multicolumn{4}{|c|}{20} \\
\hline DL:UL ratio & \multicolumn{4}{|c|}{$3: 1$} \\
\hline Subcarrier permutation mode & \multicolumn{4}{|c|}{ DL FUSC, DL PUSC, DL AMC } \\
\hline \multicolumn{5}{|c|}{ Base Station(BS) Parameters } \\
\hline Carrier Frequency $\left(f_{c}\right)$ & \multicolumn{4}{|c|}{$2.3 \mathrm{GHz}$} \\
\hline Total TX power & \multicolumn{4}{|c|}{$20 \mathrm{dBm}$} \\
\hline Antenna Gain & \multicolumn{4}{|c|}{$15 \mathrm{dBi}$} \\
\hline Modulation Scheme & \multicolumn{4}{|c|}{ QPSK, 16QAM, 64QAM } \\
\hline Coding Rate & \multicolumn{4}{|c|}{$1 / 2,3 / 4,5 / 6$} \\
\hline Coding Type & \multicolumn{4}{|c|}{ Convolutional Turbo Coding (CTC) } \\
\hline Antenna Configuration & \multicolumn{4}{|c|}{ SISO } \\
\hline \multicolumn{5}{|c|}{ Mobile Station(MS) Parameters } \\
\hline Antenna Parameters & \multicolumn{4}{|c|}{ Omni directional } \\
\hline Gain & \multicolumn{4}{|c|}{ OdBi } \\
\hline Noise Figure & \multicolumn{4}{|c|}{$7 \mathrm{~dB}$} \\
\hline Velocity $(v)$ & \multicolumn{4}{|c|}{$3 \mathrm{Km} /$ hour } \\
\hline MAX Delay Spread & \multicolumn{4}{|c|}{3700 nsec. } \\
\hline $\begin{array}{l}\text { MAX Doppler Spread }\left(f_{D}=v \times f_{c} / \text { Light }\right. \\
\text { Speed })\end{array}$ & \multicolumn{4}{|c|}{$6.38 \mathrm{~Hz}$} \\
\hline
\end{tabular}

* $n$ : Sampling factor. This parameter determines the subcarrier spacing and the useful symbol time. This value is set as follows: for channel bandwidths that are a multiple of $1.75 \mathrm{MHz}$, then $n=8 / 7$; else, for channel bandwidths that are a multiple of any of 1.25 , $1.5,2$, or $2.75 \mathrm{MHz}$, then $n=28 / 25$; else, for channel bandwidths not otherwise specified, then $n=8 / 7$. 


\section{5- System Performance Analysis:}

\subsection{Operating Distance Analysis:}

The maximum base station-mobile station separation distance (D) can be calculated according to equation (2) which represents path loss model suggested for pedestrian test environment [12]:

$$
P_{L}(d B)=40 \log _{10} D+30 \log _{10} f_{c}+49
$$

where:-

$P_{L}(d B)$ :Average path loss between the base station (TX) and mobile station (RX)

$D$ :BS-MS separation distance $(\mathrm{km})$

$f_{c}$ :Carrier frequency $\mathrm{MHz}$

Path loss is calculated according to the link budget equation expressed in equation (3):

$$
P_{L}(d B)=P_{T}(d B m)+G_{B S}(d B)+G_{M S}(d B)-P_{R}(d B m)
$$

where:-

$P_{T}$ : Base station transmit power.

$G_{M S}$ : Mobile station antenna gain.

$G_{B S}$ : Base station antenna gain.

$P_{R}$ : The received power computed according to equation (4).

$P_{R}(d B m)=S N R(d B)+$ ThermalNoise $(d B m)+$ NoiseFigure $(d B)$

where:-

SNR : Received Signal-to-Noise Ratio.

Noise Figure: Receiver noise figure (at MS).

Thermal Noise $=K \times T \times B$

$K=$ Boltzman's constant $\left(1.38 \times 10^{-23} \mathrm{~J} / \mathrm{K}\right)$.

$T=$ Receiver temperature $(290 \mathrm{~K})$.

$B=$ Receiver bandwidth (10 MHz).

\subsection{Throughput Analysis:}

Throughput is a measure of the efficiency of a network expressed as the data transfer rate of useful information. The peak physical layer error-free $\mathrm{DL}$ throughput $\left(\mathrm{R}_{\text {peak }}\right)$ per base station for a certain link-speed can be calculated using equation (5)[14]:

$R_{\text {peak }}=N_{D} N_{b} R_{F E C} / T_{s}$.

where $N_{D}$ is the number of assigned data subcarriers to the users, $N_{b}$ is the number ofmapped bits per subcarrier, $R_{F E C}$ is the FEC (Forward Error Correction) coding rate and $T_{S}$ is the OFDMA symbol duration. The actual link throughput per base station is estimated from the Packet Error Rate(PER) according to equation (6)[14]:

$R=R_{\text {peak }}(1-P E R)$

Where PER depends on the channel characteristics, SNR at the receiver, and the selected link speed according to the adaptive modulation and coding process.

\section{6- Simulation Results:}

The simulation model for the DL mobile WiMAX system consists of a base station as the transmitter followed by the ITU-R Ped. B channel with pedestrian speed of $(3 \mathrm{Km} / \mathrm{h})$ then a mobile station as the receiver. The PER calculated at the receiver by demodulating the incoming signal (which is faded and corrupted with noise) and is compared with the original transmitted data after adding a proper delay to it. 
i. System Performance in Terms of Optimum Cyclic Prefix (CP):

In this section, the PER performance vs. average SNR, for mobile WiMAX system, that described previously (for the three types downlink subcarrier permutation modes (FUSC, PUSC, AMC)), will be evaluated under various $\mathrm{CP}$ values. In order to determine the optimum $\mathrm{CP}$ value that eliminates the effects of ISI and reduces the power loss and the throughput degradation due to the overhead of this CP on the OFDMA symbol duration.

The link speed 16QAM scheme and Convolutional Turbo Coding (CTC) with coding rate $1 / 2$, are chosen in this simulation, because they are considered as a moderate value for PER performance, throughput and operating distance as compared to other link speeds. The simulation results in figures(7), (8) for PUSC, AMC show that the $\mathrm{CP}=T_{b} / 16$ length better than the other CP lengths $\left(T_{b} / 4, T_{b} / 8, T_{b} / 32\right)$ in PER reduction at higher values of SNR, this results agrees with [7], [11] which were dealt with PUSC permutation mode in their analysis and simulation. The simulation results for FUSC figure (9) show that the $\mathrm{CP}=T_{b} / 4$ slightly better than the $\mathrm{CP}=T_{b} / 16$ and other $\mathrm{CP}$ lengths (at $\mathrm{PER}=1 \times 10^{-3}$, the $\mathrm{CP}=T_{b} / 4$ better than the $\mathrm{CP}=T_{b} / 16$ by $0.3 \mathrm{~dB}$ in $\mathrm{SNR}$ ). In order to take the effects of $\mathrm{CP}$ length on other important system parameters into account, the results of peak DL throughput per base station for the three subcarrier permutation modes, with the SNR loss $\left(S N R_{\text {loss }}\right)$ which is calculated according to equation(7)[1,2,7]are listed in table (4).

$S N R_{\text {loss }}=-10 \log _{10}\left(1-T_{g} /\left(T_{g}+T_{b}\right)\right)$

where $T_{g}, T_{b}$ values are listed in table (3)

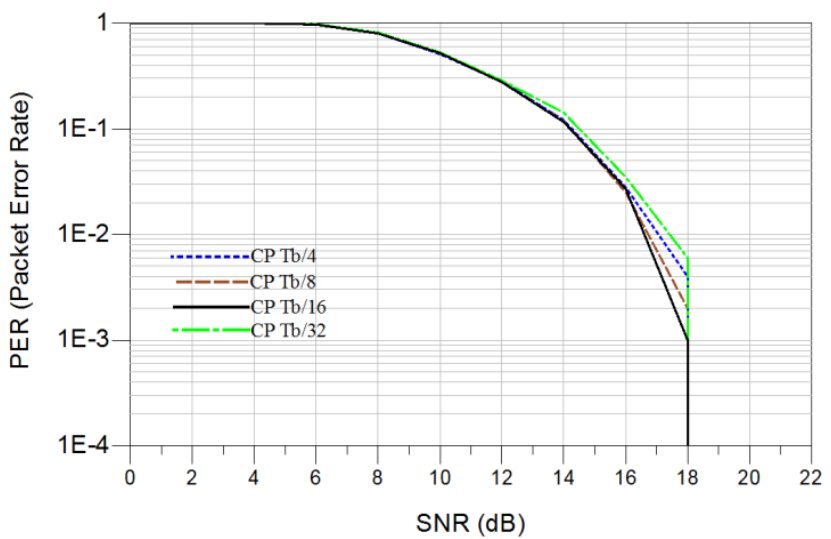

Figure (7): PER vs. SNR for PUSC mode different $\mathrm{CP}$ values

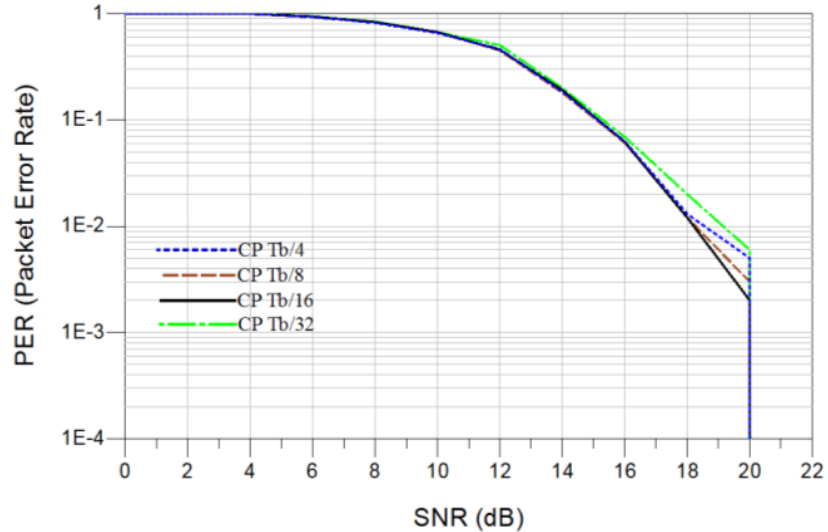

Figure (8): PER vs. SNR for AMC mode different $\mathrm{CP}$ values

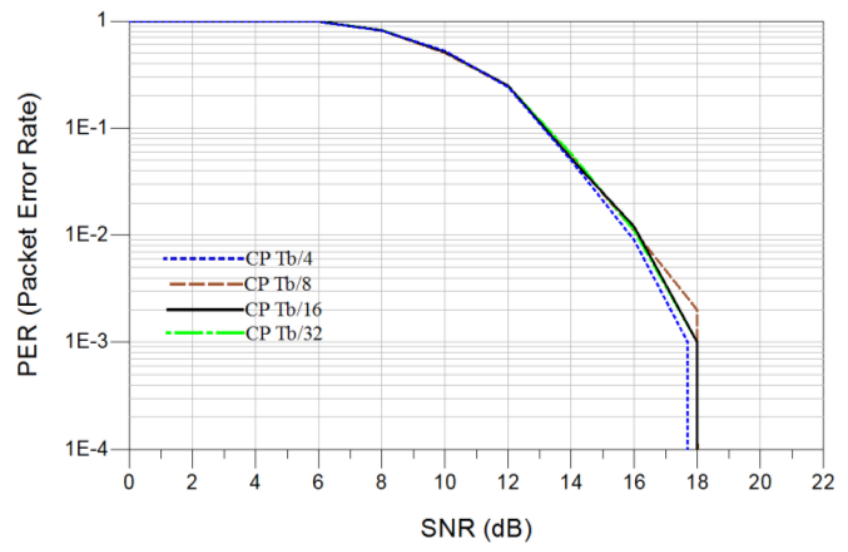

Figure (9): PER vs. SNR for FUSC mode - different CP values 
Table (4) shows the maximum throughput obtained, using equation (5), for all possible link speeds with different CP lengths. It is clear that the results of FUSC and AMC are identical, also they give better throughput than the PUSC that is because they use the same number of data subcarriers in the DL transmission, which are greater than that used in the PUSC as illustrated in table (1).

Table (4): Peak obtained throughput and SNR loss in terms of various subcarrier permutation modes, link speeds and $\mathrm{CP}$ values

\begin{tabular}{|c|c|c|c|c|c|c|c|c|c|}
\hline \multirow{2}{*}{$\begin{array}{c}\text { Subcarrier } \\
\text { permutation } \\
\text { mode }\end{array}$} & \multirow{2}{*}{$\begin{array}{c}\mathrm{CP} \\
\text { lengths }\end{array}$} & \multicolumn{7}{|c|}{ Peak Obtained Throughput (Mbps) } & \multirow{2}{*}{$\begin{array}{c}S N R_{\text {loss }}(\mathrm{d} \\
\mathrm{B})\end{array}$} \\
\hline & & $\begin{array}{c}\text { QPSK } \\
1 / 2\end{array}$ & $\begin{array}{c}\text { QPSK } \\
3 / 4\end{array}$ & $\begin{array}{c}\text { 16QAM } \\
1 / 2\end{array}$ & $\begin{array}{c}\text { 16QAM } \\
3 / 4\end{array}$ & $\begin{array}{c}\text { 64QAM } \\
1 / 2\end{array}$ & $\begin{array}{c}\text { 64QAM } \\
3 / 4\end{array}$ & $\begin{array}{l}\text { 64QA } \\
\text { M5/6 }\end{array}$ & \\
\hline \multirow[t]{4}{*}{ FUSC, AMC } & $T_{b} / 4$ & 6.72 & 10.08 & 13.44 & 20.16 & 20.16 & 30.24 & 33.6 & $\begin{array}{c}0.9691001 \\
3\end{array}$ \\
\hline & $\bar{T} T_{b} / 8$ & 7.466 & 11.2 & 14.93 & 22.4 & 22.4 & 33.6 & 37.3 & $\begin{array}{c}0.5115252 \\
24\end{array}$ \\
\hline & $T_{b} / 16$ & 7.905 & 11.85 & 15.81 & 23.71 & 23.71 & 35.57 & 39.5 & $\begin{array}{c}0.2632893 \\
87\end{array}$ \\
\hline & $T_{b} / 32$ & 8.145 & 12.21 & 16.29 & 24.43 & 24.43 & 36.65 & 40.7 & $\begin{array}{c}0.1336396 \\
16\end{array}$ \\
\hline \multirow[t]{4}{*}{ PUSC } & $T_{b} / 4$ & 6.3 & 9.45 & 12.6 & 18.9 & 18.9 & 28.35 & 31.5 & $\begin{array}{c}0.9691001 \\
3\end{array}$ \\
\hline & $T_{b} / 8$ & 7 & 10.5 & 14 & 21 & 21 & 31.5 & 35 & $\begin{array}{c}0.5115252 \\
24\end{array}$ \\
\hline & $T_{b} / 16$ & 7.41 & 11.11 & 14.82 & 22.23 & 22.23 & 33.35 & 37.05 & $\begin{array}{c}0.2632893 \\
87 \\
\end{array}$ \\
\hline & $T_{b} / 32$ & 7.63 & 11.45 & 15.27 & 22.9 & 22.9 & 34.36 & 38.18 & $\begin{array}{c}0.1336396 \\
16\end{array}$ \\
\hline
\end{tabular}

In addition, it is clear that the peak throughput obtained increases with the decrease of the CP lengths for all link speeds, this is because the CP overhead on the symbol duration is reduced; for the same reason mentioned. It can also be shown that the effect of reducing the CP length leads to reduction in the SNR loss, which is an important factor at the receiver (MS).

It is important to mention that the CP length must be selected by the primarily negotiation done between the base station and mobile station, and the CP length cannot be changed during the frame transmission DL or UL, because changing the CP would force all the Mobile Stations to resynchronize to the Base Station [10].

Depending on the simulation results, the optimum value for the $\mathrm{CP}$ length in the PUSC and AMC permutation modes will be $\mathrm{CP}=T_{b} / 16=5.71 \mu \mathrm{sec}$. In the FUSC, though there is a $0.3 \mathrm{~dB}$ improvement by the $\mathrm{CP}=T_{b} / 4$ over the $\mathrm{CP}=T_{b} / 16$, we must take into account the enhanced performance due to the increased throughput and reduced $S N R_{\text {loss }}$ by the $\mathrm{CP}=T_{b} / 16$ over the $\mathrm{CP}=T_{b} / 4$. Then, the value of $\mathrm{CP}=T_{b} / 16=5.71 \mu \mathrm{sec}$ length (which excessed the maximum r.m.s delay spread $(3.7 \mu \mathrm{sec}$.) of the ITU-R Ped. B channel) will be selected as an optimum $\mathrm{CP}$ length in the three permutation modes and it will be dependent in the further simulation of this paper.

\section{ii. System Performance in Terms of PER:}

Figure (10) shows PER performance vs. SNR for various subcarrier permutation modes and link speeds, as the coding rate is higher. The PER, performance degraded due to reduced ability for errors correction in multipath fading channel. 


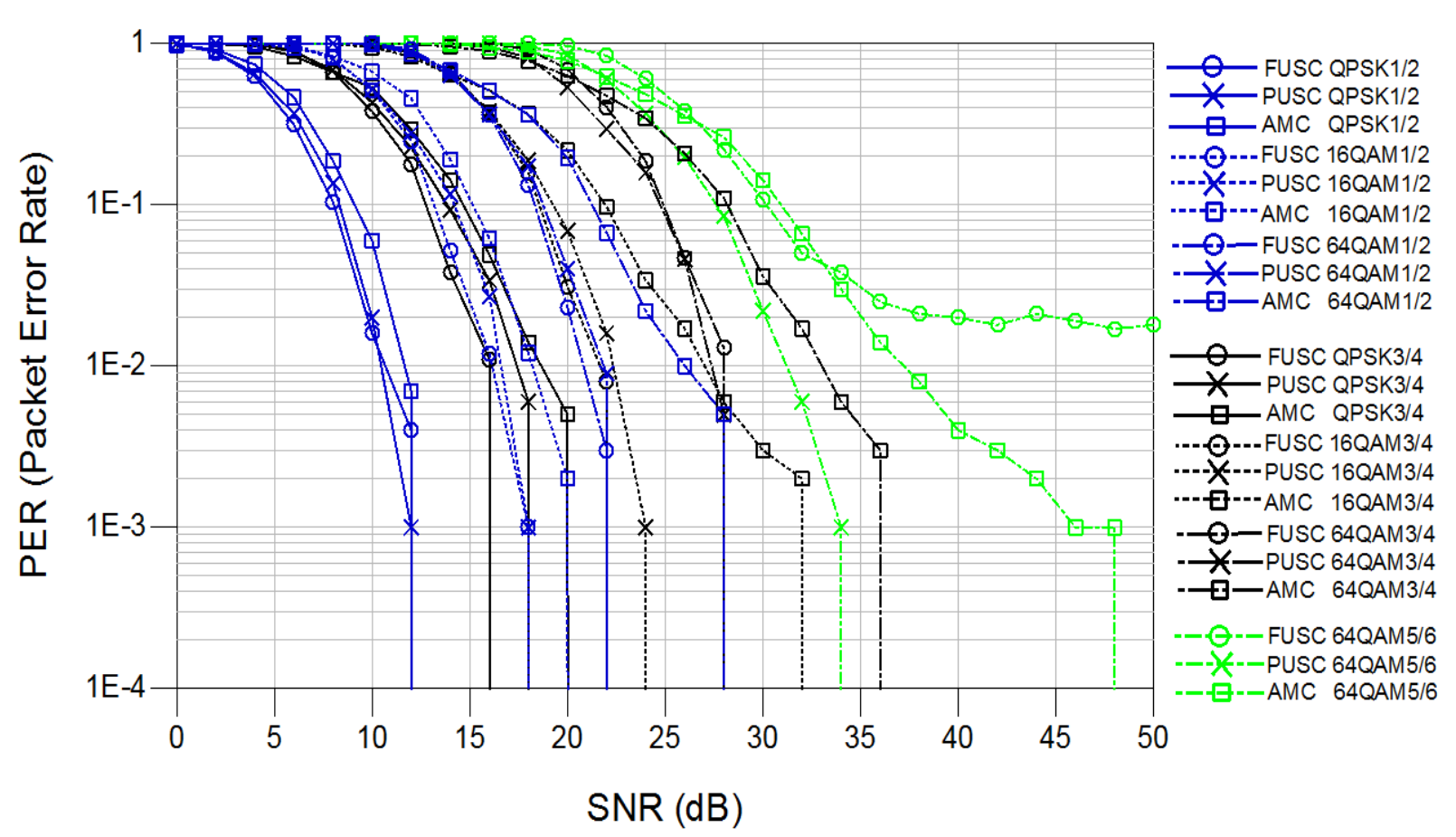

Figure (10): PER vs. SNR for various subcarrier permutation modes and link sneeds

The important observation that can be noticed from the simulation results in figure (10), is that there is a slight difference in the PER performance between different subcarrier permutation modes at the lower link speeds (QPSK1/2, QPSK3/4, 16QAM1/2). Which means that when the MS's are far from the BS $(\mathrm{SNR}=12-22 \mathrm{~dB})$ there is no significant improvement in system performance (max. improvements $=1.2 \mathrm{~dB}$ at $\mathrm{PER}=1 \times 10^{-2}$ ) between the three permutation modes (FUSC, PUSC, AMC). On the other side, it can be seen that at the higher link speeds (16QAM3/4, 64QAM1/2, 64QAM3/4), there are a significant performance improvement in the distributed subcarrier permutation modes (FUSC, PUSC) over the adjacent subcarrier permutation mode (AMC). This means at close distance ( $\mathrm{SNR}=22-36 \mathrm{~dB}$ ) from BS, there are a significant effect on the performance due to the selected subcarrier permutation mode (max. improvements $5 \mathrm{~dB}$ at $\mathrm{PER}=1 \times 10^{-2}$ ). At the 64QAM5/6, we can see that the PUSC outperform the AMC, but the FUSC produces the worst PER performance with minimum $P E R=1.8 \times 10^{-2}$ in spite of increasing the SNR until $50 \mathrm{~dB}$. This means that when the MS's are very close to the BS (SNR $\geq 32 \mathrm{~dB}$ ) it is recommended to select the PUSC permutation mode in order to achieve the best performance in PER and operating distance. So according to the received SNR and the assumed threshold PER, it is important to select a proper subcarrier permutation mode to achieve the best performance of the mobile WiMAX system.

It is also clear that the FUSC mode outperform the other two (PUSC and AMC) modes due to the high frequency diversity across the total bandwidth. Which lead to a better performance in multipath fading channel environment except at link speed 64QAM5/6 where the higher coding rate $(5 / 6)$ with the high frequency diversity lead to difficulty in correcting errors. Also, it is noticed that there are some useful cases that may be used for performance improvements in mobile WiMAX system by achieving better throughput and operating distance. The 64QAM3/4 link speed for FUSC and PUSC modes outperform the 16QAM3/4 link speed for AMC at a PER $\leq 6 \times 10^{-3}$. The 64QAM5/6 link speed for PUSC mode outperforms the 64QAM3/4 link speed for AMC at a PER $\leq 1.97 \times 10^{-1}$. If these cases are taken into account at the BS when decided to switch from a lower link speed to a higher link speed 
depending on the received SNR during the adaptive modulation and coding process, it will improve the overall performance of the system.

\section{iii. System Performance in Terms of DL Throughput and Operating Distance:}

Based on the simulation results in (ii) and equation (6), figure (11) and(12)show the comparison results for DL throughput with respect to SNR and operating distance respectively. As mentioned in the earlier, the mobile WiMAX promising high data rates and affluent multimedia services; UDP is more suitable for multimedia traffic. In this throughput and operating distance simulation, the UDP-based application is assumed to be video streaming. The PER thresholds for UDP-based applications is 1\% PER, which is considered the highest PER acceptable for UDP-based applications; any PER in excess of this value is assumed to be too severe to maintain a practical data link [15].

Figures (11), (12) show that the QPSK1/2 link speed with any one of the permutation modes (FUSC, PUSC, AMC) achieves the maximum range $(\mathrm{D}=0.18 \mathrm{Km})$ from the $\mathrm{BS}$, but with minimum DL throughput per $\mathrm{BS}(\mathrm{R}=7.874 \mathrm{Mbps}$ at $\mathrm{FUSC}$ and $\mathrm{AMC}, \mathrm{R}=7.404 \mathrm{Mbps}$ at PUSC) to maintain the assumed link connection at $1 \%$ PER. On the other hand the 64QAM5/6 with AMC permutation mode achieves the maximum DL throughput per BS ( $\mathrm{R}=$ $39.21 \mathrm{Mbps})$ with a maximum range $(\mathrm{D}=0.04 \mathrm{Km})$ to maintain the assumed link connection at $1 \%$ PER. Also, it can be seen that the higher modulation and coding rate 64QAM5/6 at FUSC mode cannot achieve the PER thresholds for UDP-based applications at 1\% PER because this link speed produces a significant PER which is higher than the assumed threshold PER. It is also clear that the peak obtained throughput for FUSC and AMC modes are equal and outperform the PUSC mode (this is illustrated in table (4)). Now for better performance for the mobile WiMAX system in the three permutation modes at a certain DL sub frame, we recommended to follow the step stairs (curves intersections) shown in figure (11), (12) to switch from link speed to another depending on the SNR in figure (11) and on the Distance in figure (12). Recall that the simulation is done based on threshold link connection of $1 \%$ PER.

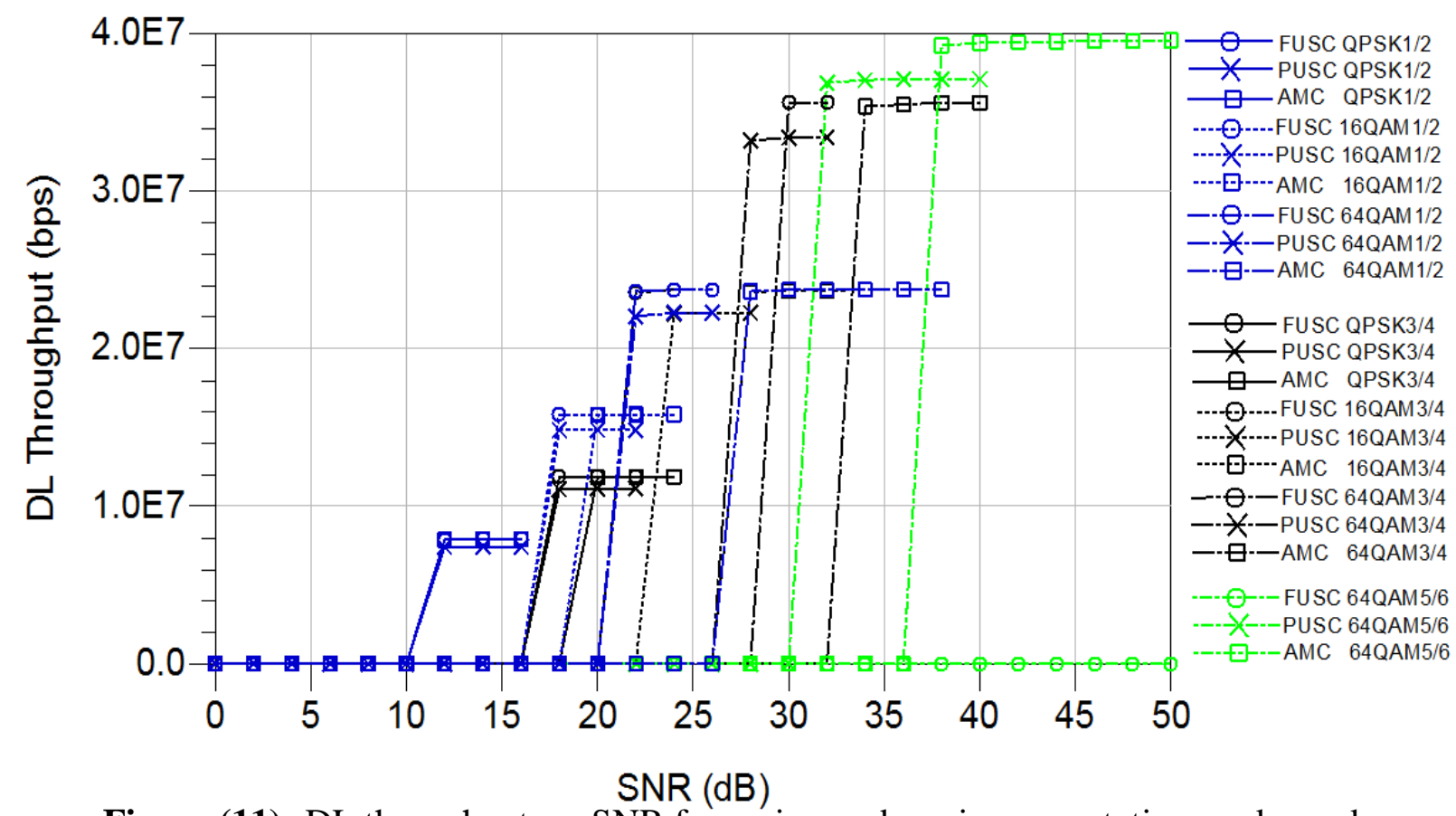

Figure (11): DL throughput vs. SNR for various subcarrier permutation modes and link speeds $-1 \%$ PER threshold 


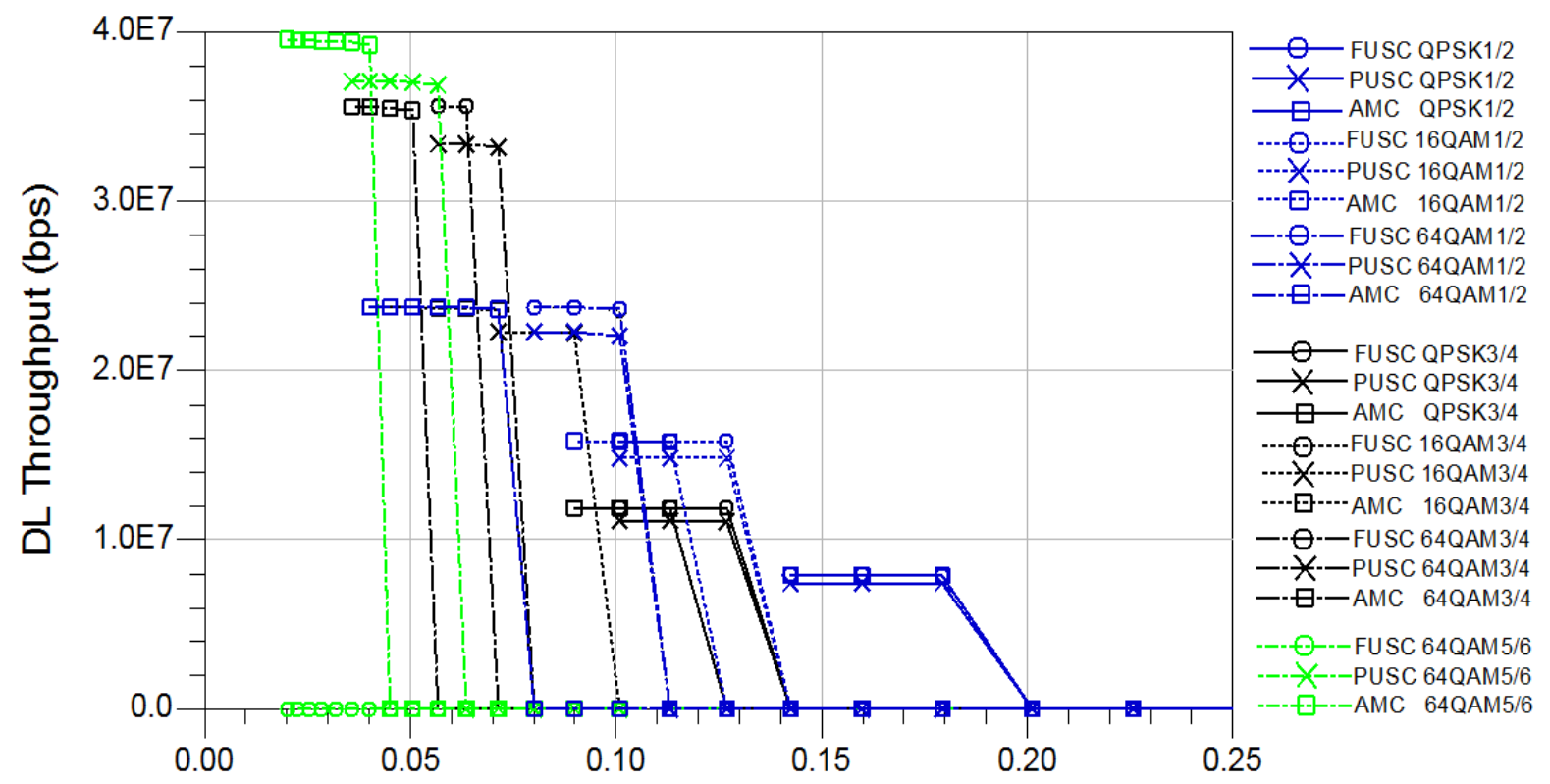

Distance $(\mathrm{Km})$

Figure (12): DL throughput vs. operating distance for various subcarrier permutation modes and link speeds $-1 \%$ PER threshold

In order to evaluate the overall simulation results for the mobile WiMAX system, table (5) shows the minimum required SNR to obtain the maximum distance with the maximum achieved throughput to maintain the link connection at $1 \%$ PER for different link speeds and permutation modes.

Table (5): Min. required SNR, Max. achieved operating distance and Max. obtained throughput for various subcarrier permutation modes and link speeds

\begin{tabular}{|c|c|c|c|c|c|c|c|c|}
\hline $\begin{array}{c}\text { Subcarrier } \\
\text { permutatio } \\
\text { n mode }\end{array}$ & Parameters & $\begin{array}{c}\text { QPSK } \\
1 / 2\end{array}$ & $\begin{array}{c}\text { QPSK } \\
3 / 4\end{array}$ & $\begin{array}{c}\text { 16QAM } \\
1 / 2\end{array}$ & $\begin{array}{c}\text { 16QAM } \\
3 / 4\end{array}$ & $\begin{array}{c}\text { 64QAM } \\
1 / 2\end{array}$ & $\begin{array}{c}\text { 64QAM } \\
3 / 4\end{array}$ & $\begin{array}{c}\text { 64QAM } \\
5 / 6\end{array}$ \\
\hline \multirow[t]{3}{*}{ FUSC } & $\begin{array}{l}\text { Min. required SNR } \\
(\mathrm{dB})\end{array}$ & 12 & 18 & 18 & 22 & 22 & 30 & $\mathrm{NA}$ \\
\hline & $\begin{array}{l}\text { Max. achieved } \\
\text { Distance }(\mathrm{Km})\end{array}$ & 0.179 & 0.127 & 0.127 & 0.101 & 0.101 & 0.064 & NA \\
\hline & $\begin{array}{c}\text { Max. obtained } \\
\text { Throughput (Mbps) }\end{array}$ & 7.874 & 11.86 & 15.8 & 23.53 & 23.65 & 35.58 & NA \\
\hline \multirow[t]{3}{*}{ PUSC } & $\begin{array}{l}\text { Min. required SNR } \\
(\mathrm{dB})\end{array}$ & 12 & 18 & 18 & 24 & 22 & 28 & 32 \\
\hline & $\begin{array}{l}\text { Max. achieved } \\
\text { Distance }(\mathrm{Km})\end{array}$ & 0.179 & 0.127 & 0.127 & 0.090 & 0.101 & 0.071 & 0.057 \\
\hline & $\begin{array}{c}\text { Max. obtained } \\
\text { Throughput (Mbps) }\end{array}$ & 7.404 & 11.05 & 14.81 & 22.21 & 22.04 & 33.19 & 36.84 \\
\hline \multirow[t]{3}{*}{ AMC } & $\begin{array}{l}\text { Min. required SNR } \\
(\mathrm{dB})\end{array}$ & 12 & 20 & 20 & 28 & 28 & 34 & 38 \\
\hline & $\begin{array}{l}\text { Max. achieved } \\
\text { Distance }(\mathrm{Km})\end{array}$ & 0.179 & 0.113 & 0.113 & 0.071 & 0.071 & 0.051 & 0.040 \\
\hline & $\begin{array}{c}\text { Max. obtained } \\
\text { Throughput (Mbps) }\end{array}$ & 7.874 & 11.8 & 15.78 & 23.58 & 23.6 & 35.36 & 39.21 \\
\hline
\end{tabular}

It is obvious from table (5) that the simulation results for the maximum throughput obtained is very close to the peak throughput obtained, at $\mathrm{CP}=1 / 16$ which is calculated in table (4). 


\section{7- Conclusion:}

In this paper, the downlink performance analysis of distributed, and adjacent subcarrier permutation modes, used in mobile WiMAX system operating in ITU-R Ped.. B channel is investigated for different parameters of physical layer such as modulation, coding schemes and the optimum value of the cyclic prefix.

The results show that the optimum cyclic prefix valve for the FUSC, PUSC and AMC subcarrier permutation modes that achieve the best performance is $T_{b} / 16$ of the OFDMA symbol time.

The results also show a slight differences in the PER performance between the FUSC, PUSC and AMC subcarrier permutation modes at the lower modulation and coding schemes (QPSK1/2, QPSK3/4, 16QAM1/2). Which means that when the MS's are far from the BS there is no significant improvement in system performance in selecting any one of the three permutation modes (FUSC, PUSC, AMC). At the higher modulation and coding schemes (16QAM3/4, 64QAM1/2, 64QAM3/4), there are a significant performance improvements of the distributed subcarrier permutation modes (FUSC, PUSC) over the adjacent subcarrier permutation mode (AMC). This means at close distance from BS, it is recommended to use the FUSC subcarrier permutation mode due to its higher throughput rather than the PUSC subcarrier permutation mode at the same operating distance as shown in figure (12). At the 64QAM5/6, it can be seen that the PUSC outperform the AMC. Though FUSC is also a type of distributed subcarrier permutation, its performance in terms of PER performance is worse than that of PUSC and AMC, which produces minimum $P E R=1.8 \times 10^{-2}$ in spite of increasing the SNR until 50dB. This means that when the MS's are very close to the BS it is recommended to select the PUSC permutation mode in order to achieve the best performance in PER and operating distance.

\section{8- References:}

[1] IEEE Std 802.16 ${ }^{\mathrm{TM}}$-2009, Revision of IEEE Std 802.16-2004 "IEEE Standard for Local and metropolitan area networks Part 16: Air Interface for Broadband Wireless Access Systems"

[2] IEEE Std 802.16e-2005, IEEE Std 802. 2004/Cor 1-2005, "IEEE Standard for Local and metropolitan area networks, Part 16: Air Interface for Fixed and Mobile Broadband Wireless Access Systems, Amendment 2: Physical and Medium Access Control Layers for Combined Fixed and Mobile Operation in Licensed Bands and Corrigendum 1."

[3]J. G. Andrews, A. Ghosh, R. Muhamed, "Fundamentals of WiMAX Understanding Broadband Wireless Networking", Prentice Hall Press, 2007.

[4]H.Yaghoobi, Intel Communications Group, Intel Corporation, "Scalable OFDMA Physical Layer in IEEE 802.16 WirelessMAN", Intel Technology Journal, Volume 8, Issue 3, 2004.

[5]WiMAX Forum, "Mobile WiMAX - Part 1: A Technical Overview and Performance Evaluation", August 2006.

[6]V.Bykovnikov, "The Advantages of SOFDMA for WiMAX", 2005 Intel Corporation.

[7] W.E.Osman, T.AbdRahman, "Optimization of Guard Time Length for Mobile WiMAX System over Multipath Channel”, Proceedings of IMECS 2008, vol. 2, 19-21 March, Hong Kong.

[8] Uma ShankerJha, Ramjee Prasad, "OFDM Towards Fixed and Mobile Broadband Wireless Access", Artech House, 2007.

[9] M. Maqbool, M. Coupechoux, and P. Godlewski, "Subcarrier permutation types in ieee 802.16e,” ENST (Telecom Paris), Technical Report, 2008. 
[10] LoutfiNuaymi, "WiMAX: Technology for Broadband Wireless Access", John Wiley \& Sons, 2007.

[11] R. M.Colda, T. Palade, E. Puşchiţă and I. Vermeşan, "Transmission Performance Evaluation of Mobile WiMAX Pedestrian Environments", 17th Telecommunications forum TELFOR 2009 Serbia, Belgrade, November 24-26, 2009.

[12] ITU Document, "Rec.ITU-R M.1225 - Guidelines for Evaluation of Radio Transmission Technologies for IMT-2000”, ITU-R, 1997.

[13] WiMAX Forum, "WiMAX System Evaluation Methodology", Version 2.1, July 2008.

[14] M. Tran, D. Halls, A. Nix, A. Doufexi, M. Beach, "Mobile WiMAX: MIMO performance analysis from a quality of service (QoS) viewpoint", IEEE Wireless Comunications and Networking Conference (WCNC) Proceedings, 2009. 